\title{
Health and social determinants of health in Vietnam: local evidence and international implications
}

\author{
Hoang Van Minh $\cdot$ Hung Nguyen-Viet
}

Received: 28 November 2016/ Accepted: 30 November 2016/Published online: 9 December 2016

(c) Swiss School of Public Health (SSPH+) 2016

Vietnam has made significant progress relating to the health status of its people and has achieved several healthrelated Millennium Development Goals such as the reduction of infant, under-five, and maternal mortality. These improvements in health outcomes have resulted from the developments in social, economic and health care conditions in Vietnam. However, Vietnam still faces many challenges regarding the protection and promotion of human health, as the country is now facing the burdens of diseases, adverse impacts on the environment, aging trends, the inadequate capacity of health system, and problems of inequities in health and health care.

The increase in non-communicable problems in Vietnam was demonstrated by the longitudinal research of LeThi et al. (2016). Students who were victimized often, as well as those who were classified as highly involved as both victims and bullies, during one or both surveys, showed significantly higher levels of depression, psychological distress, and suicidal ideation than other students. Persistent and frequent bullying was strongly linked with poor mental health for males and females (Le et al. 2016).

This editorial is part of the supplement "Health and social determinants of health in Vietnam: local evidence and international implications".

H. Van Minh $\cdot$ H. Nguyen-Viet $(\square)$

Hanoi University of Public Health, Hanoi, Vietnam

e-mail: h.nguyen@cgiar.org

H. Van Minh

e-mail: hvm@huph.edu.vn

H. Nguyen-Viet

International Livestock Research Institute (ILRI),

Hanoi, Vietnam
There are also significant environmental health issues in Vietnam. Tuyet-Hanh et al. (2016) traced the presence of legal livestock feed antibiotics to pork meat, liver and kidney in wet markets in Nghe An and Hung Yen provinces. The risk associated with microbial contamination in pork is significant. Dang-Xuan et al. (2016) used quantitative microbial risk assessment (QMRA) to estimate an $17.7 \%$ annual incidence rate of salmonellosis in humans who consumed boiled pork in urban Hung Yen province. Le-Thi et al. (2016) also used QMRA to show that the annual diarrhea risks caused by exposure to three pathogens (E. coli $\mathrm{O} 157: \mathrm{H} 7$, G. lamblia, and C. parvum) in biogas effluent were much higher than the acceptable risk proposed by WHO (reference level of 10-3 as annual risk) for wastewater reuse in agriculture. Vu-Van et al. (2016) showed that the addition of locally available materials (lime, and rice husk) to human feaces during storage can help deactivate Ascaris lumbricoides eggs more quickly to achieve a safe reuse of human feaces as fertilizers after 3 months. Winkler et al. (2016) employed a health impact assessment (HIA) approach that indicated, from a public health perspective, the reuse of liquid and solid waste as a means to recover water and nutrients and to produce energy, has considerable potential for health benefits if appropriately managed and tailored to local contexts.

To address health problems and improve the health status of Vietnamese populations, evidence-based public health interventions are needed. Dang et al. (2016) showed that pre-treatment factors such as height, weight, Hepatitis $B$ vaccination and platelet infusion made a significant difference between the treatment failure or success of HIV patients. In addition, the starting age of treatment, CD4 percentage, and opportunistic infection were found to significantly predict treatment outcome, implying the importance of clinical markers in the treatment response. 
Bich and Cuong (2016) completed a quasi-experimental, pre-test/post-test, non-equivalent control group design study, and concluded that breastfeeding, education materials, and counseling services yielded improvements in fathers' knowledge, attitude and involvement in supporting exclusive breastfeeding.

Quality of prenatal health care services is an important issue in Vietnam. Ha et al. (2016) conducted a cross sectional study on the prenatal diagnostic (PND) services provided in Vietnam and found there to be a variety of services among centers and, specifically, that the number of services provided by three PND centres in the study group was below the target set by the Ministry of Health. As well, there are still limited capacities in human resource, facilities and equipment in PND centers. Duong et al. (2016) also conducted a PDN cross-sectional study, and reported in 2016 that only $65.4 \%$ of pregnant women were satisfied with the PND services they used.

Equity-oriented reform has also been identified as a key orientation of the health system in Vietnam. Inequities in health care across different groups of population still exist. Kien et al. (2016) used 2000, 2006, 2011, and 2014 data from the Vietnam Multiple Indicator Cluster Surveys to investigate children-related socioeconomic factors. Belonging to ethnic minority groups, having mothers with lower education, and belonging to the poorest group, meant these children were less likely to receive measles vaccines, although their vaccine coverage rates did increase with time. Research by Nguyen and Wilson (2016) found the insurance coverage of the near-poor in selected communities was only $20.3 \%$, where the enrollment in the health insurance scheme was significantly associated with poor health status, good knowledge of health insurance, interest in health insurance, and the perceived cost of the insurance premium.

The findings from this cluster of research provide additional evidence on health and social determinants of health in Vietnam. We hope that policy makers, managers, health care services staff and other health system stakeholders in Vietnam and in other countries will find this cluster of scientific papers useful.
Acknowledgements We gratefully acknowledge Julie Hood, a Veterinarian Without Borders volunteer, for an English language review of this editorial.

\section{References}

Bich TH, Cuong NM (2016) Changes in knowledge, attitude and involvement of fathers in supporting exclusive breastfeeding: a community-based intervention study in a rural area of Vietnam. Int J Public Health. doi:10.1007/s00038-016-0882-0

Dang MD et al (2016) Clinical characteristics of pediatric HIV-1 patients treated with first-line anti-retroviral therapy in Vietnam: a nested case-control study. Int J Public Health $1-10$ (in press)

Dang-Xuan S et al (2016) Quantitative risk assessment of human salmonellosis in the smallholder pig value chains in urban of Vietnam. Int J Public Health. doi:10.1007/s00038-016-0921-x

Duong DTT, Huong NTT, Ha BTT (2016) Patterns of the utilization of prenatal diagnosis services among pregnant women, their satisfaction and its associated factors in Viet Nam. Int J Public Health. doi:10.1007/s00038-016-0925-6

Ha BTT, Huong NTT, Duong DTT (2016) Prenatal diagnostic services in three regional centers in Vietnam. Int J Public Health 1-7. doi:10.1007/s00038-016-0897-6

Kien VD, Van Minh H, Giang KB, Mai VQ, Tuan NT, Quam MB (2016) Trends in childhood measles vaccination highlight socioeconomic inequalities in Vietnam. Int $\mathrm{J}$ Public Health 1-9. doi:10.1007/s00038-016-0899-4

Le HTH, Nguyen HT, Campbell MA, Gatton ML, Tran NT, Dunne MP (2016) Longitudinal associations between bullying and mental health among adolescents in Vietnam. Int J Public Health 1-11. doi:10.1007/s00038-016-0915-8

Le-Thi T, Pham-Duc P, Zurbrugg C, Luu-Quoc T, Nguyen-Mai H, Vu-Van T, Nguyen-Viet H (2016) Diarrhea risks by exposure to livestock waste in Vietnam using quantitative microbial risk assessment. Int J Public Health. doi:10.1007/s00038-016-0917-6

Nguyen TD, Wilson A (2016) Coverage of health insurance among the near-poor in rural Vietnam and associated factors. Int $\mathrm{J}$ Public Health 1-11. doi:10.1007/s00038-016-0911-z

Tuyet-Hanh TT et al (2016) Exposure assessment of chemical hazards in pork meat, liver, and kidney, and health impact implication in Hung Yen and Nghe An provinces, Vietnam. Int J Public Health. doi:10.1007/s00038-016-0912-y

Vu-Van $\mathrm{T}$ et al (2016) Ascaris lumbricoides egg die-off in an experimental excreta storage system and public health implication in Vietnam. Int J Public Health. doi:10.1007/s00038-016-0920-y

Winkler MS, Fuhrimann S, Pham-Duc P, Cisse G, Utzinger J, Nguyen-Viet H (2016) Assessing potential health impacts of waste recovery and reuse business models in Hanoi, Vietnam. Int J Public Health. doi:10.1007/s00038-016-0877-x 